

Arqueología

\title{
Tecnología de caza en la Quebrada de Antofalla, Departamento Antofagasta de la Sierra, Catamarca.
}

\author{
Enrique Moreno
}

CONICET, Escuela de Arqueología, Universidad Nacional de Catamarca. E-mail: enalmor@yahoo.com

\begin{abstract}
Resumen
Las prácticas de cacería de camélidos silvestres han sido relevantes para la reproducción de las poblaciones humanas en los Andes Centro-Sur durante los últimos 12000 años aproximadamente. Para la materialización de esta práctica, son relevantes las armas utilizadas para poder dar muerte a las presas pretendidas. Una alternativa para interpretar el tipo de arma es a través de la información obtenida del análisis de las puntas de proyectil líticas registradas mediantes metodologías disciplinarias. Como caso de estudio utilizamos los datos obtenidos de la prospección intensiva y sistemática desarrollada en la quebrada de Antofalla, ubicada en el Departamento Antofagasta de la Sierra, Provincia de Catamarca, Argentina. Para evaluar las características de esta tecnología nos centraremos en tres grandes aspectos: las características tecno-morfológicas de estos instrumentos, la asignación a distintos tipos de armas y sus posibles asignaciones cronológicas, los espacios donde fueron registrados estos especímenes, en relación con las características de los paisajes cinegéticos.
\end{abstract}

Palabras clave: Tecnología; cacería; camélidos; armas; paisaje.

Hunting technology in the Antofalla Valley, Antofagasta de la Sierra Department, Catamarca.

\begin{abstract}
The hunting practice of wild camelids has been relevant for the reproduction of human populations in the South Central Andes for the last 12000 years. An important aspect for the materialization of this practice, are the weapons used in order to kill the alleged preys. In this paper we shall introduce the different weapons used by human populations, mainly, through the information obtained from the analysis of of lithic projectile points. As a case study we will retake the information obtained from the intensive and systematic prospection developed at the Antofalla ravine, located in the Departamento Antofagasta de la Sierra, Provincia de Catamarca. In order to evaluate the features of this technology, we will focus on three aspects: the techno-morphological characteristics of these instruments, the allocation to different types of weapons and their possible chronological implications and the spaces where these specimens were recorded, in relation with the characteristics of the hunting landscapes.
\end{abstract}

Key words: Technology; hunting; camelids; weapons; landscape.

Desde las primeras ocupaciones humanas en los Andes Centro-Sur, el aprovechamiento de los camélidos sudamericanos ha sido un factor relevante para la reproducción social. Entre las diferentes estrategias de apropiación de estos animales, la cacería ha sido la de más larga duración, realizándose casi hasta la actualidad. Variadas han sido las fuentes de datos para analizar esta actividad, aunque se centraron en los conjuntos faunísticos (Aschero et al. 1991, Cartajena 1994, 2003, Elkin 1996, Haber 2006, López 2003, 2006, Martínez et al. 2010, Mondini y Elkin 2006, Mondini et al. 2011, Moreno y Revuelta 2010, Olivera 1997; Revuelta 2005, Yacobaccio y Madero 1992, Yacobaccio et al. 1994) la tecnología utilizada para dar muerte y procesar las presas obtenidas (Aschero y Martínez 2001, Escola 2000, Hocsman 2006, Martínez 2003, 2005, Moreno 2010a, 2010b, Nuñez
1983; Nuñez y Santoro 1988; Pintar 1996, 2008, Ratto 2003) y el análisis de los paisajes donde se realizaron las cacerías (Aschero y Martínez 2001, Haber 2003a, 2003b, 2006, 2007; Martínez 2003, Moreno 2009, 2010b, Ratto 2003) ${ }^{1}$. También se han desarrollado aportes que buscan la articulación de diferentes fuentes de datos para la comprensión de las estrategias de caza y su relevancia en coyunturales contextos históricos y geográficos (Aschero

\footnotetext{
1 Otras fuentes de datos son relevantes para la comprensión de las prácticas de caza, como por ejemplo, restos orgánicos de las armas (astiles, intermediarios, etc.), análisis de fibras de los especímenes abatidos y aportes de disciplinas como la etología, la etnohistoria, la historia oral (Ambrosetti 1976, Cieza de León [1553] 1984, Custred 1979, Dedenbach 1990, Millones y Schaedel 1980, Moreno 2010b, Núñez et al. 2005, Pintar 2008, Polo de Ondegardo [1571] 1990, Ratto 2003, Reigadas 1992, 1994, Rodríguez 1999, Yacobaccio et al. 2007)
} 
y Martínez 2001, Haber 2003b, Martínez et al. 2010, Moreno 2009, 2010b, Ratto 2003). En este sentido, entendemos que para llevar a cabo la cacería una serie de aspectos son necesarios, entre los que podemos nombrar: las armas utilizadas, las características del paisaje y las posibles modificaciones introducidas por los cazadores (por ejemplo, la construcción de parapetos), la etología de las posibles presas, las relaciones sociales entre los cazadores (por ejemplo, el número de cazadores que participan) y otros aspectos como el viento o la luz solar (Aschero y Martínez 2001, Haber 2003a, 2003b, 2009, Martínez 2003, Moreno 2009, 2010b, Ratto 2003). En el caso particular de este trabajo nos interesa desarrollar el primero de estos aspectos, es decir, las armas que fueron utilizadas por los cazadores para intentar dar muerte a las presas. Esto se apoya en la importancia que posee la tecnología de armas a la hora de considerar las posibles estrategias de caza. Para ello nos centramos en los datos obtenidos de las prospecciones intensivas llevadas a cabo en la quebrada de Antofalla, Departamento Antofagasta de la Sierra, Provincia de Catamarca, Argentina (Figura 1). Durante estos trabajos de campo recolectamos un conjunto de puntas de proyectil que conformarán el universo analizado aquí. Este conjunto será evaluado desde diferentes enfoques para evaluar aspectos tales como las materias primas aprovechadas, las características tecnológicas y los diseños de estos instrumentos, así como también las armas utilizadas y la cronología relativa de ellas y profundizar su articulación con el paisaje.

\section{Las armas en el contexto andino}

La arqueología se ha preocupado a lo largo de la historia por las prácticas de caza, siendo uno de los ejes principales para su análisis el estudio de la tecnología de armas, haciendo hincapié en las puntas de proyectil dada sus posibilidades de conservación frente a otros materiales (por ejemplo, las maderas utilizadas como astiles) ${ }^{2}$. Las puntas de proyectil han centrado la discusión en diferentes aspectos tanto tecnológicos (diseños, materias primas), así como lo que se refiere a su eficacia o performance hasta factores sociales como identidad, diferencias de género o etarias en los contextos sociales (Bleed 1986, Churchill 1993, Cotterell y Kamminga 1990, Dobres 2000, Edmonds 1995, Gero 1989, Moreno 2005, 2006, Pfaffenberger 1992, Ratto 1994, Wiessner 1983). En este marco, en lo que se refiere a las investigaciones relacionadas con las prácticas de caza en el contexto andino, el análisis de las puntas de proyectil ha permitido discutir aspectos relevantes a la hora de interpretar las estrategias o técnicas de caza. Es por ello que uno de los principales esfuerzos de estas investigaciones se encuentra centrado en la identificación

\footnotetext{
2 En diferentes contextos han sido recuperados astiles, arcos, lanzaderas, etc (Aguerre et al. 1973, Fernández Distel 1986, Gambier 1985, Martínez 2003, 2005, Pintar 2008). Sin embargo las puntas de proyectil son las piezas que mayores posibilidades de conservación poseen y sobre los que se han centrado los análisis.
}



Figura 1. Mapa de ubicación de la quebrada de Antofalla, Catamarca, Argentina.

Figure 1. Map showing the location of the Antofalla valley, Catamarca, Argentina.

de las diferentes armas utilizadas para la realización de cacerías. De esta manera, conociendo el arma y por lo tanto características como la performance, la efectividad, el poder de penetración o la distancia de tiro, permite la reconstrucción de distintas estrategias de cacería (De Souza 2004, Martínez 2003, Moreno 2010b, Ratto 1994, 2003). Así, la información obtenida del análisis de las puntas de proyectil y de la asignación a distintos tipos de armas, sumado a aspectos relevantes del paisaje (abras, pasos, recurso hídrico), las relaciones entre cazadores y la etología de las presas conforman el marco a través del cual se interpretan las estrategias de cacería y se evalúa la relevancia de estos instrumentos en esta práctica de larga duración (Aschero y Martínez 2001, Martínez 2003, 2005, Pintar 2008, Ratto 2003)

\section{Área de estudio}

La quebrada de Antofalla se encuentra ubicada en el sector noroeste del Departamento Antofagasta de la Sierra, en lo que se define como Puna Salada o Meridional (Núñez y Santoro 1988, Olivera 1992, Troll [1931]1980). Ésta es una de las quebradas que desciende hacia el Salar de Antofalla, en dirección NO-SE siguiendo el curso de agua que se origina en dos ojos de agua ubicados a aproximadamente 4100 y 3900 msnm y que en su parte más baja forma un cono de deyección con una altura aproximada de 3400 msnm donde se pierde en el salar. Esta particularidad del régimen hídrico forma un paisaje de vega alrededor del curso de agua, siendo árido el resto del paisaje (Figura 2). Las características principales de esta área son: clima árido, fuertes variaciones de temperatura entre el día y la noche, baja cobertura vegetal, topografía quebrada, con fuertes pendientes y alturas elevadas. Las precipitaciones son principalmente nivales, predominando 




Figura 2. Vista general del paisaje de la Quebrada de Antofalla, donde resaltan las laderas de importante pendiente y el fondo de valle conformado por la vega. Al fondo se ubica el Salar de Antofalla.

Figure 2. General view of the Antofalla valley landscape, The steep slopes and the marsh placed in the bottom of the valley is showed. In the back of the figure it is placed the Salar de Antofalla.

las que ocurren en los picos más elevados. Con respecto a la vegetación, el paisaje de la Puna Salada se caracteriza por la presencia de dos formas básicas de distribución: por un lado, una vegetación concentrada, conformada por vegas que se caracterizan por una cobertura de césped más o menos continuo, asociados a corrientes de agua lenta (Haber 1991). Por otro lado una vegetación dispersa conformada por tolares y pajonales (Cabrera 1957, Elkin 1992, 1996, Haber 1991, Jofré 2004, Morlans 1995). En lo que se refiere a la fauna local se basa en la presencia de camélidos, representados por llamas (Lama glama) y vicuñas (Vicugna vicugna); roedores de las familias Abrocomidae, Cavidae, Ctenomydae, Cricetidae, entre otros; carnívoros tales como el puma (Puma concolor) y el zorro (Pseudolapex sp.) y aves como el suri (Pterocnemia pennata) (Elkin 1996, García et al. 2000)

\section{La prospección en la Quebrada de Antofalla}

En esta quebrada se diseñó una prospección tendiente a recorrer la totalidad del área con el objetivo de identificar la distribución y el asentamiento de las evidencias (Criado Boado 1999). Para ello se diseñaron transectas en las que cada participante se encontraba ubicado a $40 \mathrm{~m}$ del siguiente. Esta separación permitía una intensidad que teniendo en cuenta las características ambientales locales, en donde resaltan una baja obtrusividad general, amplia visibilidad y alta abundancia y agregación, permiten una alta confiabilidad de los resultados, obteniéndose una metodología con una considerable probabilidad de registro, disminuyendo la escala temporal (Gianotti 2004, Moreno 2010b, Nance 1979). La longitud y dirección de las transectas, al igual que el número de personas que participaron en ellas fueron variando de acuerdo a las características del entorno y al área donde se ejecutaban las transectas. A través de esta metodología se llevaron a cabo un total de 660 transectas abarcándose un área de $40 \mathrm{~km}^{2}$ aproximadamente (Moreno 2010b). Un factor de control de la primera etapa de la prospección fue la realización de muestreos sistemáticos (Binford 1972). Cada $100 \mathrm{~m}$ recorridos realizamos un muestreo superficial de $1 \mathrm{~m}^{2}$ con el objeto de analizar distribuciones en el paisaje y controlar la resolución de la prospección (Haber 1999, Moreno 2010b). Para el caso de las puntas de proyectil, todos los ejemplares fueron recolectados y transportados al laboratorio para su procesamiento. En el campo tomamos las coordenadas geográficas de cada unidad de registro, las características paisajísticas del entorno directo, la asociación con otros rasgos arqueológicos y se fotografió el área.

A partir de los datos obtenidos de la prospección de la quebrada de Antofalla se registraron evidencias sobre distintos paisajes culturales y momentos históricos (Haber 2006, Haber et al. 2002, Quesada 2007), entre los que resaltan aquellos relacionados con los paisajes de cacería, los cuales han sido desarrollados en otro lugar (Moreno 2009, 2010b), enfocándonos en este trabajo en la tecnología de caza utilizada por las poblaciones humanas para la puesta en marcha de esta práctica.

\section{Las puntas de proyectil en la quebrada de Antofalla}

En la prospección intensiva realizada en Antofalla pudimos identificar un total de 263 piezas relacionadas con la manufactura de puntas de proyectil, de las cuales 71 fueron identificadas como preformas, mientras que 192 fueron asignadas a fragmentos y especímenes completos de puntas de proyectil. Las preformas, identificadas como piezas en proceso de manufactura y que fueron abandonadas en dicho proceso no serán tenidas en cuenta aquí ya que nos interesan solo aquellas piezas que nos aportan información sobre los distintos tipos de armas y su asociación con las características del paisaje para comprender las estrategias de cacería implementadas 3 .

Por lo tanto, la muestra aquí analizada se conforma de 192 puntas de proyectil de las cuales sólo 13 fueron recuperadas completas, mientras que 179 son fragmentos, en algunos casos con una representación alta de la pieza completa y en otros casos, sólo la

\footnotetext{
3 Para más información acerca de las preformas ver Moreno (2010b).
} 
presencia de fragmentos muy pequeños. Al tratarse de un conjunto superficial, la conservación del mismo es baja, observándose una alta frecuencia de piezas ventifaccionadas.

\section{Materias primas utilizadas}

La principal materia prima utilizada para la manufactura de puntas de proyectil es el basalto-andesita negro con una cantidad de 125 instrumentos $(65,5 \%$ de la muestra total de puntas de proyectil analizadas). Siguiendo en cantidad recuperamos 48 puntas de proyectil manufacturadas en obsidiana, representando el $25 \%$ de la muestra total, aunque ésta última proviene de dos canteras diferentes. Para el caso del basalto-andesita negro, hemos registrado una cantera ubicada en la desembocadura de la quebrada de Antofalla (Af0020) ${ }^{4}$. Esta materia prima presenta características que la hacen relevante en su elección para la manufactura de puntas de proyectil, ya que es local y de una calidad muy alta para la talla y para la manufactura de distintos tipos de instrumentos, como por ejemplo las puntas de proyectil (Moreno 2005, 2010b). En el caso de la obsidiana, 28 especímenes provienen de la cantera de Ona, ubicada a unos $15 \mathrm{~km}$ de la quebrada de Antofalla. Además de la cantera de Ona, otro tipo de obsidiana fue utilizado para la manufactura de otras 20 puntas de proyectil. Esta obsidiana tiene la característica de ser opaca y presentar una mejor calidad para la talla que la de Ona. Desconocemos hasta el momento la fuente de aprovisionamiento de esta materia prima. Sin embargo, en la cuenca de Archibarca, ubicada a unos $60 \mathrm{~km}$ al noroeste de la Quebrada de Antofalla, se han encontrado dispersos en el paisaje nódulos pequeños de esta materia prima (Haber 2003b) por lo que podrían haber sido obtenida allí. En resumen estas materias primas han sido seleccionadas para la manufactura del $90 \%$ de las puntas de proyectil registradas en la quebrada de Antofalla. El 10\% restante se encuentra manufacturado en otras materias primas como el ópalo, proveniente posiblemente también de la cuenca de Archibarca, donde una cantera de esta materia prima fuera identificada (Haber 2003b). Finalmente, cinco puntas de proyectil fueron manufacturadas en materias primas no identificadas, de las cuales desconocemos hasta el momento sus fuentes de aprovisionamiento.

Por lo tanto el aprovechamiento de las materias primas para la manufactura de puntas de proyectil se basa en canteras locales inmediatas para el caso del basaltoandesita negro y mediatas lejanas para la obsidiana de Ona y no locales en el caso de la obsidiana opaca y el ópalo que provendrían de la cuenca de Archibarca (sensu Hocsman 2006) cuyas características técnicas incluyen buena calidad para la talla, poder de penetración y resistencia.

\footnotetext{
4 En la figura 1 se observa la ubicación de las posibles fuentes de aprovisionamiento de las materias primas utilizadas para la manufactura de puntas de proyectil.
}

\section{Manufactura}

En términos generales, la manufactura del conjunto de puntas de proyectil aquí analizado presenta características bastante semejantes. La serie técnica ${ }^{5}$ muestra un predominio del microrretoque, siendo la técnica con la cual se manufacturaron 179 puntas de proyectil, mientras que solamente 13 fueron manufacturadas por retoque. La extensión de los lascados muestra que 75 puntas de proyectil poseen lascados extendidos, mientras que 65 presentan lascados parcialmente extendidos y 52 lascados marginales. Con respecto a la situación de los lascados, la técnica de lascado bifacial fue utilizada casi en la totalidad de los instrumentos, observándose 185 piezas con lascados sobre ambas caras, mientras que solamente 7 presentan modificaciones sobre una sola cara del instrumento. Estos datos nos indican prácticas de manufactura bastante homogéneas que implican la reducción bifacial de las formas base. La selección de estas últimas habría sido un aspecto relevante, ya que la diversidad en la extensión de los lascados, indicaría la búsqueda de formas base que no requirieran de adelgazamiento. Por último, el microrretoque como técnica prácticamente exclusiva se relacionaría con la preparación de la forma final de la punta de proyectil y la necesidad de desarrollar un diseño que tuviera las características necesarias como parte activa de un arma cuyo objetivo es herir a una futura presa.

A partir del análisis tecnológico macroscópico de las puntas de proyectil registradas durante la prospección construimos una tipología teniendo en cuenta aspectos generales de la manufactura, pero sobre todo criterios relacionados con la forma general del contorno, forma de la base y dimensiones y que en general refieren a una homogeneidad en el diseño de las puntas de proyectil. El objetivo de esta tipología es doble: por un lado generar un cúmulo de información que pudiese ser comparable con otras investigaciones realizadas en áreas cercanas; y por otro lado poder, a partir de dicha comparación construir una cronología relativa de la tecnología de caza para la quebrada de Antofalla. Así a partir del análisis de un conjunto de 99 especímenes (51,6\% de la muestra) que presentaban caracteres tecno-morfológicos que permitían la comparación entre ellos, construimos 10 tipos morfológicos y 4 especímenes morfológicos (sensu Hocsman 2006). En la tabla 1 presentamos los resultados de este análisis, con los valores correspondientes a cada tipo y sobre los que volveremos más adelante en el texto. Además en la figura 3 presentamos los diseños de los tipos identificados en el conjunto analizado.

Dado el objetivo de este trabajo, a continuación evaluaremos las características de las posibles armas

\footnotetext{
5 Para el análisis de las puntas de proyectil nos basamos en la propuesta de Aschero (1975, 1983), además de algunos aspectos relevantes para la asignación a los distintos tipos de armas, de acuerdo a los modelos utilizados (Ratto 1994, 2003, Shott 1997).
} 


\begin{tabular}{|c|c|c|c|c|c|c|c|c|c|c|c|c|c|c|}
\hline 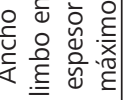 & $\stackrel{\mathfrak{N}}{\tilde{N}}$ & $\stackrel{\infty}{\stackrel{\infty}{\sim}}$ & & $\bar{\sim}$ & $\stackrel{\sim}{\check{N}}$ & $\stackrel{\text { ñ }}{\stackrel{\nu}{N}}$ & & $\stackrel{\sim}{\stackrel{N}{N}}$ & 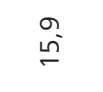 & & $\underset{\mathbb{N}}{\bar{I}}$ & & $\stackrel{m}{g}$ & $\stackrel{m}{\sim}$ \\
\hline 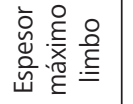 & $\stackrel{N}{\tilde{O}}$ & $\approx$ & ' & $\stackrel{⿱}{N}$ & $\bar{\sigma}$ & $\stackrel{\nexists}{\sigma}$ & & $\hat{\circ}$ & $\stackrel{6}{\circ}$ & & $\tilde{\sigma}$ & & $\stackrel{\infty}{0}^{0}$ & ${ }_{\sigma}^{m}$ \\
\hline 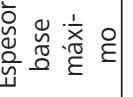 & in & $\stackrel{\substack{\infty \\
N}}{\sim}$ & ' & $\stackrel{n}{\stackrel{n}{m}}$ & $\bar{m}$ & $\hat{i}$ & $\stackrel{\leftrightarrow}{\rightarrow}$ & $\stackrel{6}{i}$ & $\stackrel{a}{i}$ & ' & $\stackrel{\infty}{m}$ & $\hat{\sigma}$ & m & $\stackrel{\sim}{\sim}$ \\
\hline 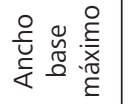 & 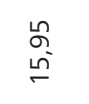 & $\begin{array}{l}\stackrel{\infty}{\infty^{-}} \\
\stackrel{\infty}{-}\end{array}$ & , & $\stackrel{\stackrel{n}{\sim}}{=}$ & $\simeq$ & $\tilde{\sigma}$ & $\stackrel{\infty}{\underset{+}{\mp}}$ & $\stackrel{m}{\stackrel{m}{n}}$ & $\bar{i}$ & & $\stackrel{+}{\stackrel{\sim}{~}}$ & $\stackrel{m}{\stackrel{m}{g}}$ & $\stackrel{m}{\sim}$ & $\stackrel{\infty}{+}$ \\
\hline 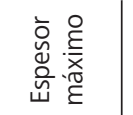 & $\stackrel{\stackrel{\sim}{=}}{=}$ & 㔯 & $\bar{\sigma}$ & $\approx$ & $\bar{\sigma}$ & $\begin{array}{l}\infty \\
\infty \\
\infty\end{array}$ & 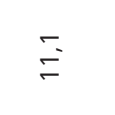 & $\bar{\sigma}$ & ín & $\bar{\sigma}$ & $m_{a}^{m}$ & $\stackrel{\infty}{\infty} \infty^{\infty}$ & $\ddot{\sigma}$ & $\sigma$ \\
\hline 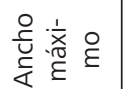 & 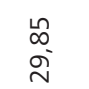 & $\stackrel{\sim}{\stackrel{\sim}{N}}$ & $\hat{i}$ & $\stackrel{+}{i}$ & $\stackrel{\mathbb{N}}{ }$ & $\begin{array}{l}\infty \\
\stackrel{\infty}{N}\end{array}$ & 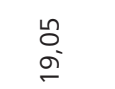 & 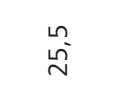 & 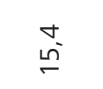 & $\begin{array}{l}m \\
\stackrel{m}{\sim} \\
\stackrel{v}{N}\end{array}$ & $\stackrel{m}{m}$ & $\stackrel{m}{\text { Dे }}$ & $\stackrel{+}{m}$ & 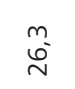 \\
\hline 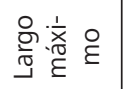 & 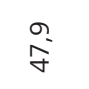 & $\begin{array}{l}\stackrel{n}{n} \\
\hat{n} \\
m\end{array}$ & $\stackrel{\sim}{N}$ & 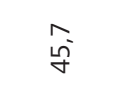 & 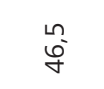 & $\frac{6}{\dot{f}}$ & $\begin{array}{l}\ell_{0} \\
\infty \\
\infty\end{array}$ & $\stackrel{\infty}{\text { Ni }}$ & 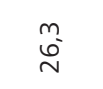 & $\stackrel{m}{m}_{-}^{\frac{n}{6}}$ & $\underset{\infty}{\infty}$ & $\underset{⿱ 亠 凶}{\dot{q}}$ & 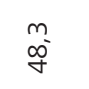 & $\stackrel{\sim}{\stackrel{f}{H}}$ \\
\hline 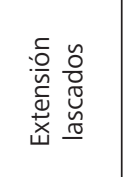 & 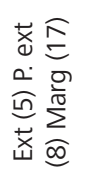 & 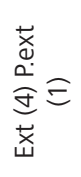 & 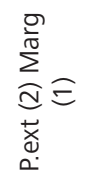 & 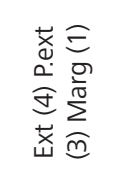 & 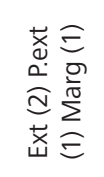 & 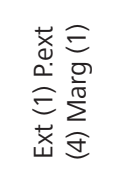 &  &  & 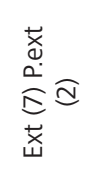 &  & $\begin{array}{l}\bar{\pi} \\
\stackrel{\overline{0}}{\overline{0}} \\
\sum_{\Sigma}^{\bar{\pi}}\end{array}$ & 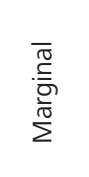 &  &  \\
\hline 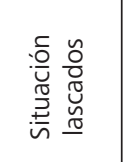 & 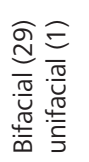 & 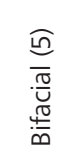 & 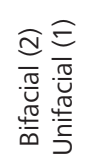 & 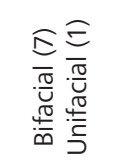 & 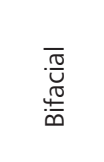 & $\begin{array}{l}\frac{\bar{\sigma}}{\bar{\sigma}} \\
\stackrel{\Phi}{\overline{0}}\end{array}$ & 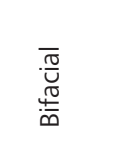 & 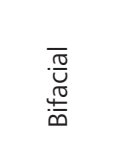 & 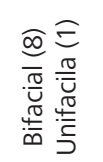 & 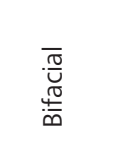 & 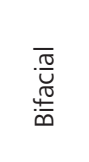 &  & $\begin{array}{l}\overline{\bar{\sigma}} \\
\stackrel{\bar{\Phi}}{\bar{\Phi}} \\
\Phi\end{array}$ & 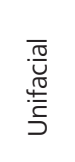 \\
\hline 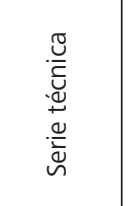 & 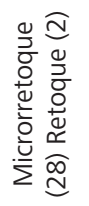 & 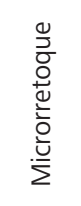 & 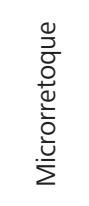 & 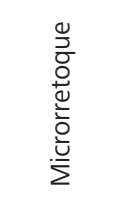 & 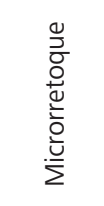 & 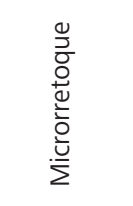 & 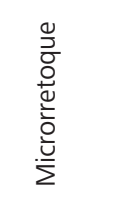 & 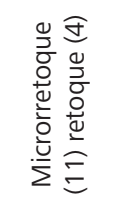 & 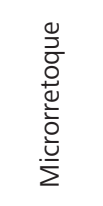 & 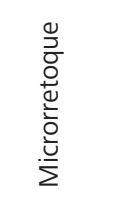 & $\begin{array}{l}\frac{0}{0} \\
\frac{0}{0} \\
\dddot{0}\end{array}$ & 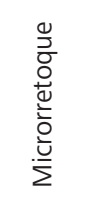 & 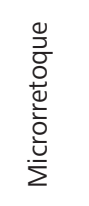 & 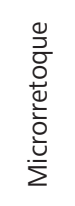 \\
\hline 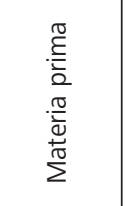 & 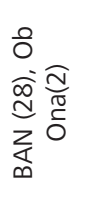 & 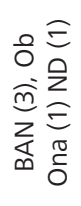 & 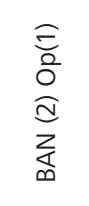 & 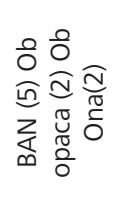 & 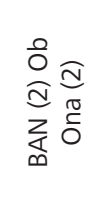 & 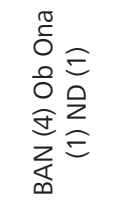 & 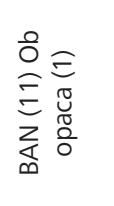 & 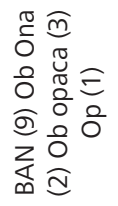 & 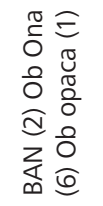 & 杯 & 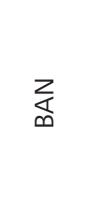 & \ał & 之 & 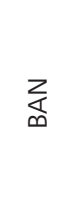 \\
\hline 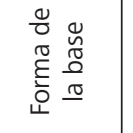 & 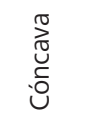 & $\underset{\mathscr{E}}{\stackrel{\mathbb{E}}{\mathscr{E}}}$ & 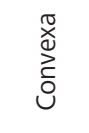 & $\underset{\mathbb{U}}{\mathbb{U}}$ & 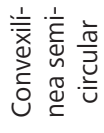 & : & 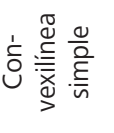 & ن & 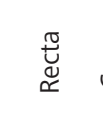 & 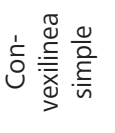 & 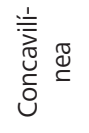 & 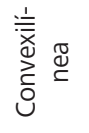 & $\underset{\mathscr{\varpi}}{\mathbb{\widetilde { g }}}$ & 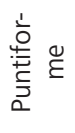 \\
\hline 苋 & 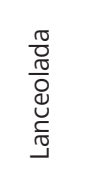 & 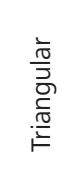 & 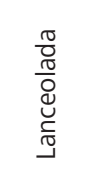 & 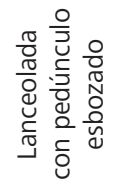 & 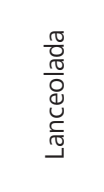 & 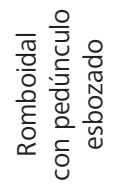 & 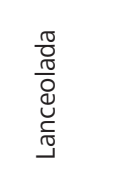 & 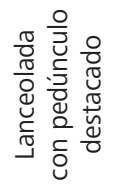 & 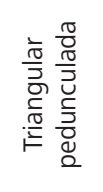 & 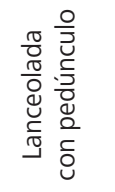 & 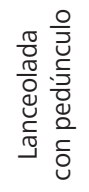 & $\begin{array}{l}\frac{\pi}{0} \\
\frac{0}{3} \\
\frac{0}{2} \\
\frac{3}{0} \\
0 \\
0\end{array}$ & 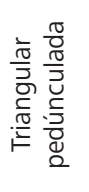 & $\begin{array}{l}\frac{\pi}{0} \\
\frac{\pi}{0} \\
\mathbb{U} \\
\frac{0}{\pi}\end{array}$ \\
\hline$z$ & $\stackrel{\circ}{m}$ & in & m & $\infty$ & $\nabla$ & 0 & $\simeq$ & $\stackrel{n}{\sim}$ & $\sigma$ & $m$ & - & - & - & - \\
\hline$\stackrel{\circ}{\circ}$ & $\frac{T}{4}$ & $\overline{\frac{\bar{T}}{4}}$ & $\underset{\frac{1}{4}}{\overline{\frac{1}{\alpha}}}$ & $\underset{\frac{1}{4}}{3}$ & $\frac{3}{4}$ & $\underset{\frac{1}{4}}{\frac{1}{4}}$ & $\sum_{\frac{1}{4}}^{\overline{1}}$ & $\dot{4} \overline{\bar{x}}$ & $\frac{x}{\frac{1}{4}}$ & 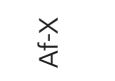 & $\underset{\frac{⿱}{4}}{4}$ & $\underset{\frac{1}{4}}{\frac{1}{4}}$ & $\underset{\frac{1}{4}}{u}$ & $\frac{9}{\frac{1}{4}}$ \\
\hline
\end{tabular}

Tabla 1: Variables utilizadas para la construcción de los tipos y los especímenes morfológicos de puntas de proyectil registradas en la quebrada de Antofalla. Las medidas utilizadas son promedios de los valores medidos en los diferentes especímenes considerados. Las medidas se encuentran expresadas en $\mathrm{mm}$.

Table 1. Variables used for the construction morphological types and specimens of the projectile points registered in the Antofalla valley. The measures are the average of the different measurements made in the specimens. The values are expressed in $\mathrm{mm}$. 

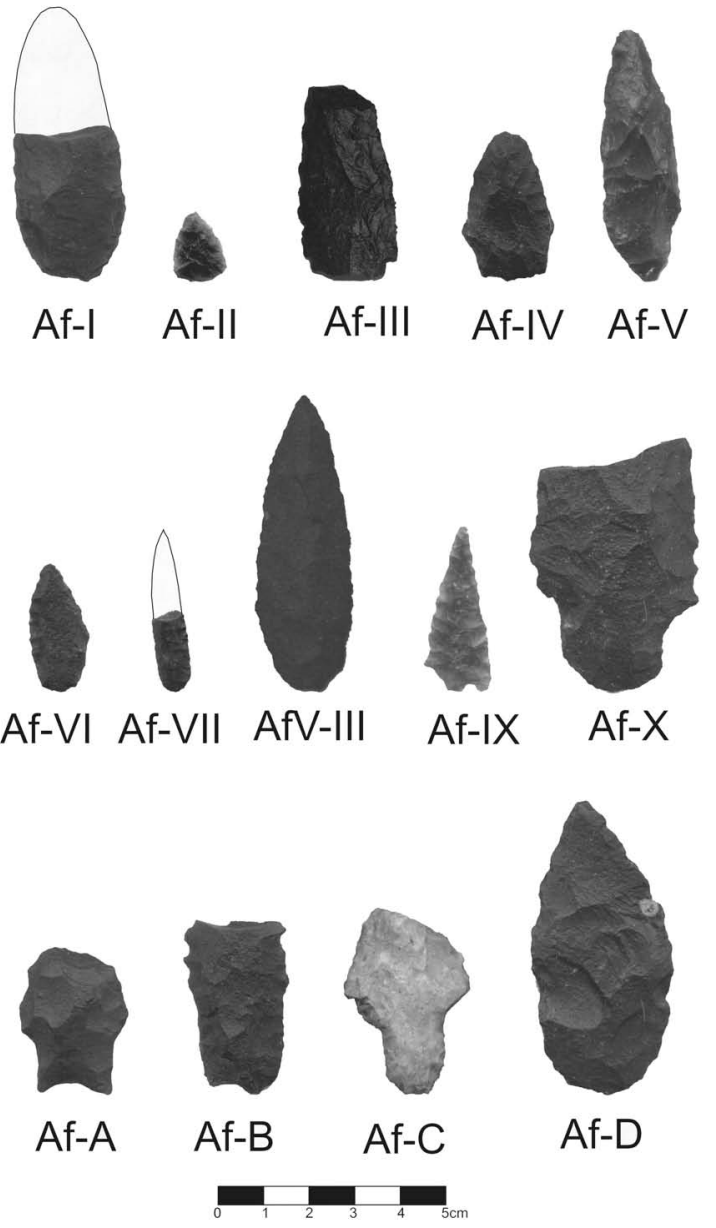

Figura 3. Tipos y especímenes morfológicos identificados en la quebrada de Antofalla. Las referencias para las materias primas son: BAN: Basaltoandesita negro; Ob Ona: Obsidiana Ona; Ob opaca: Obsidiana opaca; Op: Ópalo y ND: No diferenciada. Las referencias para la extensión de los lascados son: Ext: Extendido; P. ext: Parcialmente extendido y Marg: Marginal.

Figure 3. Morphological types and specimens identified in the Antofalla valley. The references for raw material are: BAN: Black Basalt-andesite; Ob Ona: Ona Obsidian; Ob opaca: Opaque obsidian; Op: Opal and ND: not differentiated. The references for the extension of the flakes are: Ext: extended; P. ext: Partially extended and Marg: marginal

utilizadas para la realización de cacerías, donde la diferencia observada en los diseños implicaría el uso de diferentes armas.

\section{Las armas en Antofalla}

Desde hace varios años se han desarrollado distintas perspectivas para la asignación funcional de las puntas de proyectil a los respectivos tipos de armas, teniendo en cuenta que estos instrumentos son los que poseen más posibilidades de conservación frente a los materiales orgánicos, generalmente utilizados para la manufactura de astiles, intermediarios o arcos. Variables tales como el tamaño, el peso o la forma fueron originariamente utilizadas para la asignación a los distintos tipos de armas (Fenenga 1953, Kidder 1938). Desarrollos posteriores han criticado estas estrategias analíticas
(Cattelain 1997, De Souza 2004, Hughes 1998, Ratto 1994, 2003, Shott 1997, Thomas 1978), proponiéndose diversos métodos para la asignación funcional de estos instrumentos a los tipos de armas, basándose en la comparación con especímenes etnográficos o aspectos tales como la aerodinámica o la masa de las puntas de proyectil. Para el caso particular del conjunto aquí analizado nos centraremos en dos metodologías: por un lado la propuesta de Ratto $(1994,2003)$ y por el otro la propuesta de Shott (1997) modificada a partir del método desarrollado por Thomas (1978). Esta decisión se basa en tres cuestiones principales: 1) por un lado poder obtener una mayor cantidad de puntas de proyectil que pudiesen ser asignadas a los tipos de armas dado el alto nivel de fragmentación de las piezas con la que contamos en esta investigación. Esto se relaciona con las diferentes variables tomadas por cada modelo. 2) Por otro lado, porque nos permite evaluar los límites y posibilidades de estos modelos. 3) Por último, porque ambos modelos se dirigen hacia la separación de tipos de armas distintos, por lo que creemos que sumando ambos esfuerzos podremos comprender de manera complementaria la utilización de los sistemas de armas. El modelo de Thomas (1978) y Shott (1997) permite separar entre arco y flecha y dardos de lanzadera; mientras que el de Ratto $(1994,2003)$ se basa en la separación entre puntas líticas enastiladas en sistemas técnicos con almacenamiento de energía (arco y flecha y lanza arrojadiza) y sin almacenamiento de energía (punta de arma de mano).

La primera metodología basa la asignación funcional en una serie de variables métricas: largo, ancho, espesor, ancho del pedúnculo y peso (Thomas 1978). Esta metodología fue luego profundizada por Shott (1997) quien propone como variable significativa para la asignación funcional el ancho del hombro, a partir del aumento de la muestra de puntas de proyectil asignadas a dardo de lanzadera.

Por su parte, Ratto (1994, 2003) ha generado un modelo de asignación funcional de cabezales líticos apoyándose en el análisis crítico de: (a) los equipos de caza aportados por trabajos etnográficos y experimentales, (b) la mecánica de funcionamiento de las armas en base a las leyes de la mecánica de fluidos y la trayectoria de vuelo de los proyectiles y (c) las propiedades físico-mecánicas de las materias primas líticas y vegetales. En base a estos principios se proponen una serie de aspectos relacionados a la performance del sistema técnico (Ratto 2003: 86-87):

- Superficie de refuerzo. Es aquella que soporta la fuerza de impacto o choque, en función del tipo de trayectoria de vuelo del sistema técnico.

- Aerodinámica: en función si rige o no dicha trayectoria

- Poder de penetración

- Enmangue

La metodología propuesta por Shott (1997: 93) define 
la asignación funcional en base a dos ecuaciones, una para dardo de lanzadera y la otra para arco y flecha. Aplicando estas ecuaciones a cada espécimen, aquella que otorgue el mayor valor se asigna al tipo de arma correspondiente. Las ecuaciones son las siguientes ${ }^{6}$ :

Para dardo de lanzadera:

$\mathrm{C}=0,18 \times$ largo $+0,87 \times$ ancho del hombro $+0,72 \times$ espesor $+0,21$ $x$ ancho del pedúnculo $-18,79$.

Para arco y flecha:

$\mathrm{C}=0,07 \times$ largo $+0,49 \times$ ancho del hombro $+1,28 \times$ espesor $+0,14$ ancho del pedúnculo $-8,60$.

En la tabla 2 presentamos las medidas utilizadas para el cálculo de las ecuaciones correspondientes a dardos de lanzadera y a arco y flecha, así como también los valores obtenidos a través de estas ecuaciones y por lo tanto la asignación al tipo de arma. Teniendo en cuenta el planteo de Shott (1997), sólo 32 especímenes $(16,7 \%$ de la muestra total de puntas de proyectil) pudieron ser analizados, de los cuales 8 puntas de proyectil se asociarían a arco y flecha, mientras que las restantes 24 habrían sido utilizadas como parte activa de dardos de lanzadera.

Para el caso de la metodología propuesta por Ratto (1994, 2003), el análisis se basa en una serie de factores que definimos más arriba y que los resultados de su aplicación a los especímenes registrados en la quebrada de Antofalla son presentados en la tabla 3. Solamente 14 puntas de proyectil $(7,29 \%)$ presentaban todas las variables para poder ser incorporadas al modelo propuesto. De este número, solamente una punta de proyectil no pudo ser asignada a alguno de los tipos de armas propuestos (Moreno 2010b). El resto de los especímenes

\footnotetext{
6 Las medidas para la aplicación de las ecuaciones propuestas por Shott (1997) son expresadas en milímetros.
}

\begin{tabular}{|c|c|c|c|c|c|c|c|}
\hline Identificación & Largo & Ancho & Espesor & $\begin{array}{c}\text { Ancho } \\
\text { pedúnculo }\end{array}$ & $\begin{array}{l}\text { Ecuación } \\
\text { lanzadera }\end{array}$ & $\begin{array}{c}\text { Ecuación } \\
\text { arco }\end{array}$ & Arma \\
\hline Af-1160 & 26,1 & 13,5 & 4,8 & 5,4 & 2,243 & 6,742 & Arco \\
\hline AF-1489-2000 & 70 & 35,8 & 12,2 & 25,6 & 39,116 & 33,042 & Lanzadera \\
\hline Af-1779 & 31,4 & 17,7 & 8 & 9,2 & 9,953 & 13,799 & Arco \\
\hline Af-1836 & 51,2 & 29 & 12,2 & 24,4 & 29,564 & 28,226 & Lanzadera \\
\hline AF-AFX & 45,4 & 29,3 & 8,8 & 26,5 & 26,774 & 23,909 & Lanzadera \\
\hline AF-ATF & 28,6 & 12,3 & 3,3 & 3,8 & 0,233 & 4,185 & Arco \\
\hline AF-ATV & 30,2 & 27,3 & 8,6 & 21,2 & 21,041 & 20,867 & Lanzadera \\
\hline AF-AWH & 48,7 & 23 & 8,7 & 18,4 & 20,114 & 19,791 & Lanzadera \\
\hline AF-BFP & 37,6 & 25,4 & 10 & 22,3 & 21,959 & 22,4 & Arco \\
\hline AF-BGI-2001 & 54 & 31,1 & 11,7 & 23,5 & 31,346 & 28,685 & Lanzadera \\
\hline AF-BMK & 45,7 & 28,4 & 6,7 & 24,5 & 24,113 & 20,521 & Lanzadera \\
\hline AF-BSZ & 74,1 & 29,2 & 11,1 & 24 & 32,984 & 28,463 & Lanzadera \\
\hline AF-CRB & 55,2 & 24,2 & 7,7 & 19 & 21,734 & 19,638 & Lanzadera \\
\hline AF-CRF & 48,3 & 34 & 6,4 & 15,9 & 27,431 & 21,859 & Lanzadera \\
\hline AF-CWH & 25,4 & 16,2 & 4,7 & 6,3 & 4,583 & 8,014 & Arco \\
\hline AF-CWH-2001 & 48,4 & 33,4 & 11,7 & 20,7 & 31,751 & 29,028 & Lanzadera \\
\hline AF-CWV & 50,2 & 24,3 & 10,4 & 20,4 & 23,159 & 22,989 & Lanzadera \\
\hline$A f-C X E$ & 52,5 & 34,8 & 12,2 & 26,2 & 35,222 & 31,411 & Lanzadera \\
\hline AF-CYB-2000 & 57,3 & 32,4 & 9,9 & 28,6 & 32,846 & 27,963 & Lanzadera \\
\hline AF-CYE & 46,3 & 36,3 & 11 & 22,3 & 33,728 & 29,63 & Lanzadera \\
\hline AF-CYG-2001 & 38,1 & 25,2 & 9,3 & 20,8 & 21,056 & 21,231 & Arco \\
\hline AF-CYJ-2000 & 59,8 & 36,2 & 10,3 & 15,2 & 34,076 & 28,636 & Lanzadera \\
\hline AF-CYW & 37,3 & 31,4 & 9,1 & 23,5 & 26,729 & 24,335 & Lanzadera \\
\hline AF-CZB-2000 & 51,1 & 32,3 & 12 & 19 & 31,139 & 28,824 & Lanzadera \\
\hline Af-CZB-2001 & 53,3 & 33,8 & 10,4 & 24,2 & 32,78 & 28,393 & Lanzadera \\
\hline AF-CZN & 24,2 & 29,6 & 7,4 & 21,8 & 21,224 & 20,122 & Lanzadera \\
\hline$A f-C Z V$ & 57,7 & 27,6 & 9 & 18,8 & 26,036 & 23,115 & Lanzadera \\
\hline AF-DAA & 42,2 & 24,4 & 7,6 & 17,5 & 19,181 & 18,488 & Lanzadera \\
\hline Af-DLD & 38 & 24 & 7 & 20,2 & 18,212 & 17,608 & Lanzadera \\
\hline AF-DLE & 40,8 & 20,8 & 8,2 & 18 & 16,334 & 17,464 & Arco \\
\hline Af-DUT & 33 & 21 & 7 & 9,8 & 12,518 & 14,332 & Arco \\
\hline AF-DUV & 38,6 & 30 & 9,3 & 21,6 & 25,49 & 23,73 & Lanzadera \\
\hline
\end{tabular}

Tabla 2. Datos utilizados y resultados obtenidos de la asignación funcional de las puntas de proyectil de la quebrada de Antofalla de acuerdo al modelo propuesto por Shott (1997). Las medidas se encuentran expresadas en $\mathrm{mm}$.

Table 2. Data and results from the functional assignment of the projectile points of the Antofalla valley, following the model proposed by Shott (1997). The measures are expressed in $\mathrm{mm}$. 
pudieron ser asignados a alguno de los tipos de armas propuestos por Ratto (1994, 2003).

1. Arco y flecha: Pudieron ser asignadas cinco puntas de proyectil a este tipo de arma, de las cuales cuatro fueron manufacturadas en obsidiana de Ona y una en ópalo. Estas piezas presentan índices de refuerzo bajo, salvo por dos especímenes manufacturados en materias primas vítreas, lo que se utiliza para contrarrestar una mayor fragilidad de estas materias primas. La aerodinámica es normal o perfecta en todos los casos. El ancho del pedúnculo siempre es sensiblemente menor a los 10 $\mathrm{mm}$. Un ejemplar apedunculado presenta el ancho de la base menor a los $15 \mathrm{~mm}$ por lo que fue asignada a esta categoría de arma. Finalmente el ángulo del bisel es siempre menor a $45^{\circ}$.

2. Lanzas arrojadizas: Identificamos tres puntas de proyectil correspondiente a este tipo de arma, todas manufacturadas en basalto-andesita negro. Estas piezas presentan índices de módulos de refuerzo medios o altos, y una aerodinámica en un caso imperfecta y en otra perfecta. Por último el valor del ancho de la base es superior a los $10 \mathrm{~mm}$ y el ángulo del bisel superior o igual a $45^{\circ}$. Existe una tercera punta de proyectil que podría ser asignada a este tipo de arma, ya que presenta un índice de módulo de refuerzo medio, aerodinámica imperfecta, pero con gran superficie de contacto y un ángulo igual a $45^{\circ}$. La diferencia se centra en la presencia del ancho de la base menor a $10 \mathrm{~mm}$. Sin embargo, por las demás características podría ser asignado a este tipo de arma.

3. Armas de mano: Registramos tres especímenes asignados a este tipo de arma, dos manufacturadas en basalto-andesita negro y una en obsidiana de Ona. Presentan valores bajos para el índice del módulo de refuerzo, mientras que la aeordinámica es imperfecta o no aplica, siendo la superficie de contacto mediana o grande. El ancho de la base es superior a $10 \mathrm{~mm}$ y el ángulo es siempre mayor a $45^{\circ}$, salvo en un caso que es igual a dicho valor.

4. Lanzadera: En el análisis realizado por Ratto (2003) sobre las puntas de proyectil, identificó un grupo denominado sistema especial que fue atribuido a este tipo de arma. Tomando el mismo criterio, dos puntas de proyectil fueron asignadas a este tipo de arma, ambas manufacturadas en basalto-andesita negro. Estos especímenes presentan bajo módulo de refuerzo, aerodinámica normal, valores superiores a $10 \mathrm{~mm}$ para el ancho de la base y ángulos iguales o superiores a $45^{\circ}$.

5. Finalmente un ejemplar manufacturado en basaltoandesita negro no pudo ser asignado a ningún tipo de arma. Presenta un índice medio del módulo de refuerzo, aerodinámica imperfecta, el ancho de la base sensiblemente inferior a $10 \mathrm{~mm}$ y el ángulo de $35^{\circ}$.

A partir de la articulación de ambos modelos se amplió a un total de 38 puntas de proyectil $(19,8 \%)$ las que pudieron ser asignadas a distintos tipos de armas, de las cuales 10 fueron asignadas a arco y flecha, 3 a armas de mano, 3 a lanza arrojadiza y 22 a dardos de lanzadera.

\section{La escala espacial de la cacería en la quebrada de Antofalla}

A través del registro obtenido de las prospecciones intensivas realizadas en la quebrada de Antofalla, hemos podido reconstruir las características generales del paisaje cinegético diseñado por los cazadores para propiciar el encuentro con las posibles presas (Haber y Moreno 2008; Moreno 2009, 2010b). Este paisaje se basa en la articulación entre rasgos paisajísticos relevantes, tales como abras, hondonadas o pasos con estructuras construidas con diferentes objetivos tales como ocultamiento, refugio, protección, etc. Entre las estructuras identificadas durante la prospección resaltan las trincheras, estructuras de aproximadamente 1,5 $\mathrm{m}$ de largo y una altura de 0,7 m, construidas con el objetivo de otorgar escondite a los cazadores (Figura 4). Estas estructuras se encuentran siempre construidas en sectores elevados en relación al entorno directo, asociadas a quiebres de pendientes, abras o pasos. De esta manera los cazadores obtienen un importante escondite dado por la posición topográfica a lo que se suman estas estructuras. En repetidas oportunidades, las trincheras se encuentran asociadas entre sí lo que habría permitido la realización de cacerías comunales, aunque las cacerías solitarias también podrían haber sido practicadas (Moreno 2009, 2010b). Pero además de las trincheras, otras estructuras también conforman este espacio cinegético. Entre estas
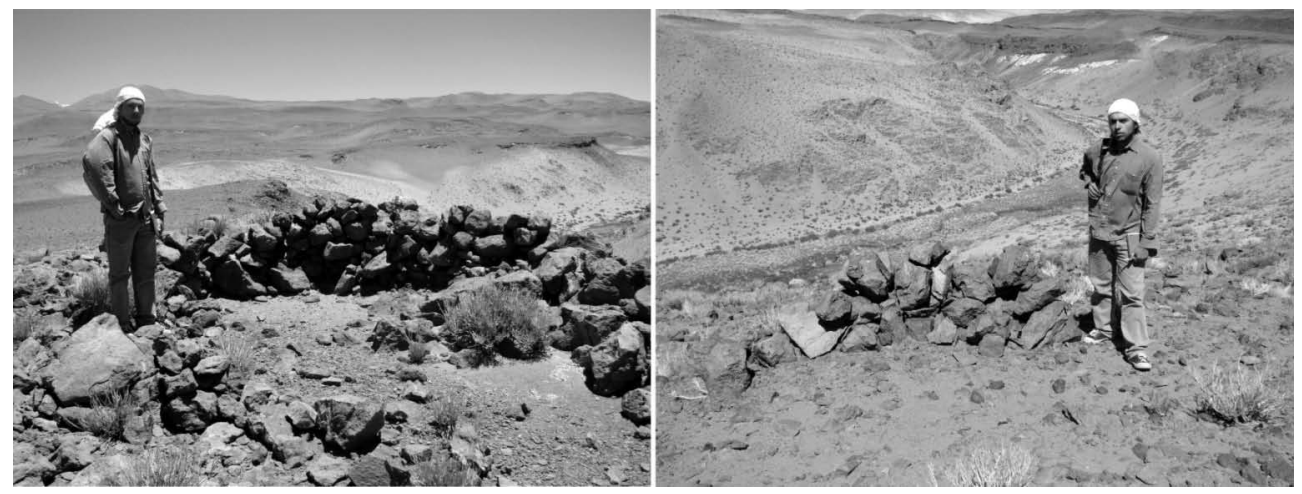

Figura 4. Trincheras utilizadas por los cazadores para esconderse de las presas. Nótese su ubicación en sectores elevados en relación a su entorno directo.

Figure 4. "Trenches" used by the hunters to hide from preys. It is relevant the placement of "trenches" in higher positions in relation of their direct environment. 


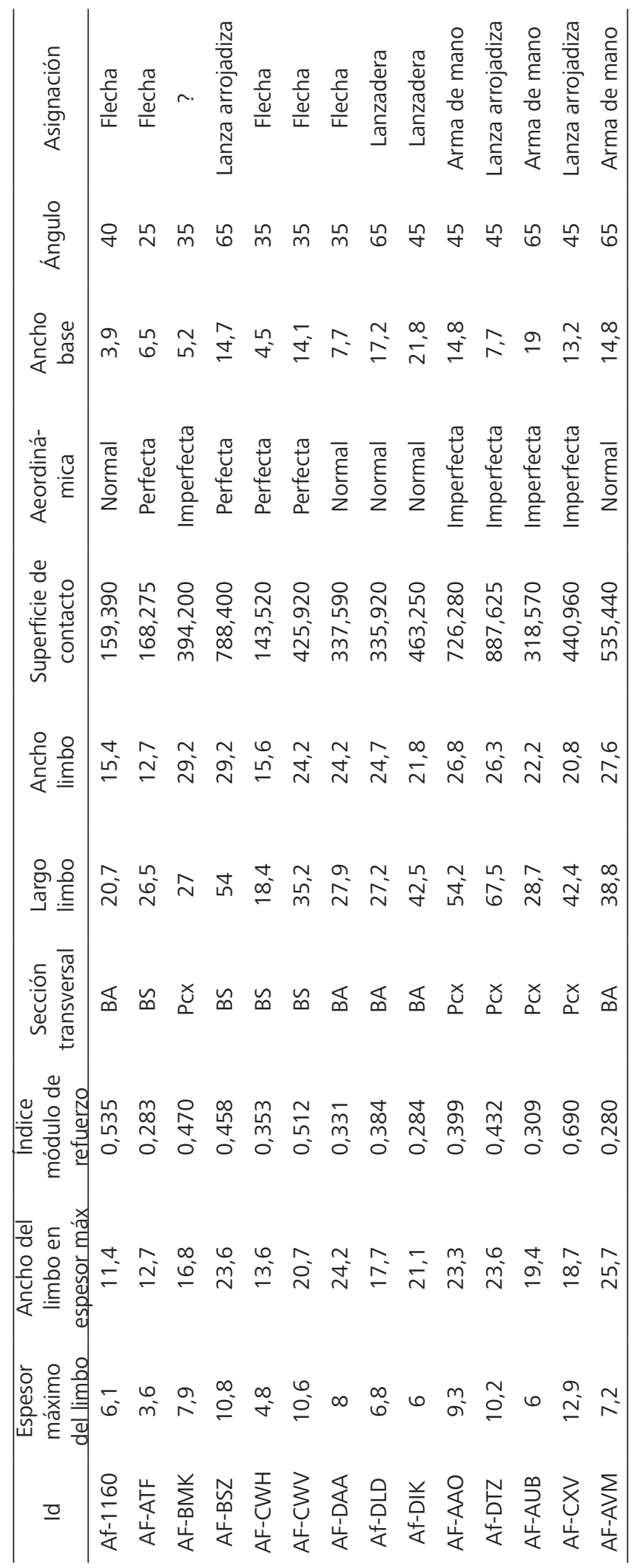

Tabla 3. Datos utilizados y resultados obtenidos de la asignación funcional de las puntas de proyectil de la quebrada de Antofalla de acuerdo al modelo propuesto por Ratto (1994, 2003). Las medidas se encuentran expresadas en $\mathrm{mm}$.

Table 3. Data and results for the functional assignment of the projectile points of the Antofalla valley, using the model proposed by Ratto (1994, 2003). The measures are expressed in $\mathrm{mm}$. estructuras registramos refugios, alineaciones, mesadas, mojones, escondites, etc. las que se articulan entre sí y con los rasgos topográficos conformando espacios en los que, interpretamos, se realizaron las cacerías (Haber y Moreno 2008; Moreno 2009, 2010b).

La construcción de estos espacios habría servido para propiciar el ocultamiento de los cazadores, aguardando el paso de los animales por sectores cercanos y de esta manera realizar el ataque buscando así aumentar las posibilidades del éxito. Hay dos cuestiones importantes que sustentan esta interpretación, por un lado la etología de las especies pretendidas y por el otro, el aspecto aquí analizado, es decir las armas con las que contaban los cazadores.

\section{La etología de las presas}

Para evaluar la etología de las posibles presas nos centramos en la información obtenida de los estudios realizados sobre vicuñas, dado por la multiplicidad de trabajos realizados sobre esta especie y por su relevancia en algunos contextos analizados en el área (Elkin 1996, Haber 2001, 2006, Moreno y Revuelta 2010, Revuelta 2005).

La organización social de la vicuña está caracterizada por la existencia de grupos familiares, tropillas de solteros y machos solitarios. En este caso nos centraremos en los grupos familiares ya que se ha evidenciado el aprovechamiento de animales de ambos sexos y diferentes grupos etarios lo que estaría implicando el aprovechamiento de grupos familiares (Elkin 1996), además de tratarse del tipo de agrupamiento que posee el movimiento diario que complementaría la información obtenida acerca del paisaje cinegético en Antofalla (Tomka 1992). Los grupos familiares se encuentran definidos por un macho dominante o relincho, que mantiene y controla el territorio, una cantidad variable de hembras adultas, hembras jóvenes mayores de un año y crías de ambos sexos menores del año (Bonacic 2005; Franklin 1982; Hoffman et al. 1983; Tomka 1992; Wheeler 2006). El relincho se encarga de proteger a la manada, estando alerta frente a posibles peligros, iniciando el escape en caso de ser necesario, mientras las hembras y crías pastan. Estos animales poseen la capacidad de escapar a gran velocidad y por escarpadas y abruptas laderas, por lo que la cacería por persecución no sería una opción viable. La forma de fijación y delimitación de los territorios se basa en la conformación de 'bosteaderos', es decir, lugares comunes de defecaciones que marcan los límites del territorio (Bonacic 2005; Tomka 1992; Wheeler 2006). Dentro de este territorio se incluyen un área alta utilizada como dormidero aprovechando para ello preferentemente roquedales que otorgan cierta protección, y áreas más bajas con pasturas y una fuente de agua. Su movimiento diario es rutinario, durmiendo en zonas altas y descendiendo durante las horas con luz 


\begin{tabular}{lllc}
\hline \multicolumn{1}{c}{ Sistema técnico } & & Alcance $(\mathrm{m})$ & Penetración $(\mathrm{cm})$ \\
\hline Dardo de lanzadera & Tiro libre: $35-74$ & Tiro efectivo: $39 \pm 5,5$ & $4-27$ \\
Lanza arrojada a mano & Tiro libre: 32 & Tiro efectivo: $7,8 \pm 2,2$ & $15 \pm 6,08$ \\
Arco y Flecha & Tiro libre: 140 & Tiro efectivo: $25,8 \pm 2,4$ & $10,75 \pm 6,56$ \\
\hline
\end{tabular}

Tabla 4. Distancias de vuelo máximas y efectivas de los distintos tipos de armas. Tomado de Ratto (2003).

Table 4. Maximum and effective flight distances of the different types of weapons. Sensu Ratto (2003).

solar a pastar y beber, retornando al crepúsculo a los roquedales utilizados como dormidero (Franklin 1982; Haber 2003a; Haber y Moreno 2008; Hoffman et al 1983; Koford 1957; Tomka 1992). Por la fisiología de estos animales, deben beber agua todos los días, por lo que este movimiento es realizado indefectiblemente de manera diaria.

Las características etológicas de las posibles presas, mostrarían la necesidad de la estructuración del paisaje para promover los encuentros y apropiarse de ciertos recursos. Es decir, son animales que poseen un movimiento rutinario, relativamente predecible, pero que a su vez perciben a los predadores mediante diversos sentidos (olfato, vista, oído), pudiendo escapar velozmente frente a prácticamente cualquier obstáculo que se le presente. En este sentido la estructuración del paisaje de cacería encontraría sentido ya que se encuentra en sectores intermedios entre los extremos de los territorios de las tropillas, permitiendo a los cazadores ocultarse, aprovechando aquellos sectores utilizados rutinariamente por los animales para alimentarse al amanecer y encontrar refugio al crepúsculo (Haber y Moreno 2008, Moreno 2010b).

\section{Tecnología y escala espacial de la cacería}

El otro aspecto relevante para comprender las prácticas de caza en la quebrada de Antofalla son las armas utilizadas. A lo largo del trabajo hemos hecho hincapié sobre este aspecto, pero resulta relevante evaluar la asociación presente entre estos instrumentos y las características de los paisajes de cacería. En este sentido, el análisis presentado más arriba acerca de la asignación de los especímenes de puntas de proyectil a distintos tipos de armas aporta una fuente de datos relevante para evaluar la asociación con las estructuras, particularmente con las trincheras y por otro lado nos permite interpretar el aprovechamiento diferencial de los espacios de cacería a nivel cronológico

Para analizar la asociación entre trincheras y puntas de proyectil tuvimos en cuenta el tipo de arma, las distancias de vuelo calculadas para cada uno de ellos (sensu Ratto 2003) y la ubicación espacial donde fueron registrados (Tabla 4). A través de un análisis de buffer $^{7}$ que permite

\footnotetext{
7 Para el análisis de buffer tuvimos en cuenta el promedio entre las distancias máximas de vuelo y la distancia efectiva para cada tipo de
}

medir la distancia desde por lo menos una trinchera, establecimos que aproximadamente el $50 \%$ de las puntas de proyectil asignadas a un tipo de arma se encontraban a distancias menores que las correspondientes a la distancia de vuelo calculada en cada caso (Moreno 2010a, 2010b). Esto permite interpretar un importante porcentaje de asociación entre las puntas de proyectil y las trincheras, apoyando la hipótesis de la funcionalidad de estas estructuras y por lo tanto de la estructuración del paisaje de cacería.

En este sentido, habíamos planteado que la tecnología de armas aportaba un factor importante para comprender la estructuración de los paisajes de cacería, ya que las mismas presentan distancias efectivas de vuelo relativamente restringidas, con un máximo de $40 \mathrm{~m}$, lo que implicaría la necesidad de obtener blancos relativamente cercanos, menores a dicha distancia para aumentar las probabilidades de éxito. Creemos entonces que la forma en que se estructuran los paisajes de cacería permite esta situación al aguardar los cazadores parapetados detrás de las trincheras a que circulen los animales por posiciones cercanas y allí comenzar el ataque.

\section{La escala temporal de la tecnología}

Las diferentes armas utilizadas para la realización de cacerías, así como también los diseños que hemos descripto más arriba permiten la construcción de una cronología relativa de esta tecnología. Ésta es establecida mediante una comparación con ejemplares que han sido recuperados en contextos con fechados radiocarbónicos del NOA y del norte chileno. Para la comparación se tomaron datos provenientes de investigaciones en la microrregión de Antofagasta de la Sierra (Escola 1987, 2000, Hocsman 2006, Martínez 2003), el norte chileno (De Souza 2004, Núñez 1983, Núñez et al. 2005) y la cuenca de Chaschuil (Ratto 2003). Esta comparación se basó, esencialmente, en la comparación de los diseños de las puntas de proyectil registrados en la quebrada de Antofalla, con los de estos sectores (Tabla 5).

Así, hemos podido identificar diseños correspondientes a diferentes momentos históricos, desde momentos tempranos (c.a. $8600 \mathrm{AP}$ ) e incluso hemos recuperado cartuchos de armas de fuego asignables al siglo $X X$ d.C. lo que nos permite interpretar una larga historia

arma (Moreno 2010a). 
relacionada con las prácticas de cacería. Con respecto a los diseños más tempranos, identificamos dos diseños que pueden ser comparables con los tipos Quebrada Seca A y Tambillo 1 asignados cronológicamente circa 8600 AP (Martínez 2003, Núñez 1983). Otro diseño identificado también a estos momentos tempranos es comparable con el diseño Peña de la Cruz A asignado circa 7270 AP (Martínez 2003).

En una imaginaria línea histórica, la mayor cantidad de especímenes de puntas de proyectil se asignan al período identificado como de "transición entre sociedades cazadoras-recolectoras a productoras de alimentos", correspondiente al lapso temporal 5150-3430 AP. Allí varios diseños pueden ser comparables a los tipos Peñas Chicas E; Peñas Chicas C; Quebrada Seca F; Peñas Chicas A de la cuenca de Antofagasta de la Sierra (Hocsman 2006).

En lo que se refiere al $1^{\circ}$ milenio de la era, hemos identificamos un diseño comparable a otras áreas, algunas cercanas como la quebrada de Tebenquiche Chico (Moreno 2005) y otras más alejadas, como los contextos que venimos comentando hasta aquí, como la microrregión de Antofagasta de la Sierra (Escola 1987, 2000, Hocsman 2006), la cuenca de Chaschuil (Ratto 2003) y el norte chileno (De Souza 2004, Núñez 1983).

Finalmente, contamos con información sobre las armas utilizadas en contextos históricos más tardíos, como son puntas de proyectil manufacturadas en material óseo y cartuchos de armas de fuego. Para el primer caso, en la quebrada de Tebenquiche Chico se recuperaron ejemplares de puntas de proyectil manufacturadas en material óseo correspondientes al período colonial temprano y que habrían formado parte de un sistema de arco y flecha (Haber 2006, Lema 2004) ${ }^{8}$. Por otro lado, en la prospección intensiva de la quebrada de Antofalla identificamos tres cartuchos de bala correspondientes a Mausser 7.65 y un ejemplar de calibre 44 (Moreno 2010b).

La comparación de los diseños de puntas de proyectil líticas, así como también la información de otros tipos de materiales y armas utilizados nos permite interpretar una larga duración en torno a las relaciones entre cazadores y camélidos silvestres, donde la cacería habría conformado una de las estrategias principales de obtención de estos últimos por parte de las poblaciones humanas (Haber 2003b, Moreno 2010b).

Ahora bien, tal como hemos presentado hasta aquí, durante las prospecciones intensivas en la quebrada de

\footnotetext{
8 En la quebrada de Tebenquiche Chico, a partir de la excavación del recinto TC2, asignado al período colonial temprano, se logró identificar en la muestra arqueofaunística un porcentaje de especies asignadas a vicuñas que alcanza el $80 \%$, mientras que las llamas alcanzan el $20 \%$. Por ello se ha interpretado la relevancia de las prácticas de caza en el contexto colonial temprano (Lema 2004, Lema y Moreno 2009, Moreno y Revuelta 2010).
}

\begin{tabular}{|c|c|c|}
\hline Tipo & Semejanza & Período \\
\hline Af-I & Peñas Chicas E & $4150-3430 \mathrm{AP}$ \\
\hline Af-II & $\begin{array}{l}\text { Quebrada Seca A - Tambi- } \\
\qquad \| \text { o-1 }\end{array}$ & 8600 AP \\
\hline Af-III & X & $x$ \\
\hline Af-IV & Peñas Chicas C & $4150-3430 A P$ \\
\hline Af-V & $x$ & $x$ \\
\hline$A f-V I$ & Quebrada Seca F & $4150-3430$ AP \\
\hline $\begin{array}{l}\text { Af- } \\
\text { VII }\end{array}$ & Peña de la Cruz A & $7270 \mathrm{AP}$ \\
\hline $\begin{array}{l}\text { Af- } \\
\text { VIII }\end{array}$ & Peñas Chicas A & $4150-3430$ AP \\
\hline Af-IX & $\begin{array}{c}\text { Registros semejantes en } \\
\text { TC1, Casa Chavez Montícu- } \\
\text { los, Chaschuil, Real Grande } \\
\text { I, Tulan-54 }\end{array}$ & $1^{\circ}$ Milenio d.C. \\
\hline Af-X & $\mathrm{X}$ & $x$ \\
\hline Af-A & Quebrada Seca B & $7350-3500 A P$ \\
\hline Af-B & $x$ & $x$ \\
\hline Af-C & $x$ & $x$ \\
\hline Af-D & Peñas Chicas 4 & $4150-3430$ AP \\
\hline
\end{tabular}

Tabla 5. Cuadro comparativo de los diseños identificados en la quebrada de Antofalla, con especímenes de otras regiones cercanas como Antofagasta de la Sierra o el Norte Chileno y su posible asignación cronológica.

Table 5. Comparative table with the designs identified in the Antofalla valley with specimens of nearby areas like Antofagasta de la Sierra or Northern Chile, and their probable chronological assignment.

Antofalla, el conjunto de puntas de proyectil recuperado implicaría el aprovechamiento de una diversidad de armas utilizadas a lo largo del tiempo con un principal objetivo, obtener camélidos silvestres. Sin embargo, estas diferencias en la tecnología podrían haber tenido consecuencias relevantes a la hora de llevar adelante las estrategias para la realización del encuentro entre cazadores y posibles presas.

Con el objetivo de evaluar la interacción entre la tecnología de caza y los paisajes en la figura 5 se presenta la distribución de las puntas de proyectil de acuerdo a su asignación funcional a los distintos tipos de armas desarrollados más arriba. Allí se puede observar un uso recursivo de los mismos paisajes en los que fueron registradas las puntas de proyectil durante el recorrido de las transectas. No se observan diferencias claras en lo que se refiere a la ubicación general de los especímenes analizados, salvo por un cartucho de bala ubicado en el sector más elevado de la quebrada donde no se registraron otros proyectiles. En el resto de los casos se identifican ejemplares de todos los tipos de armas que comparten las mismas áreas del paisaje, principalmente, es decir, aquellos sectores asociados a las trincheras y que habrían permitido el escondite de los cazadores.

En relación a los diferentes contextos históricos, la situación es semejante ya que una vez más notamos la presencia de ejemplares de puntas de proyectil correspondientes 


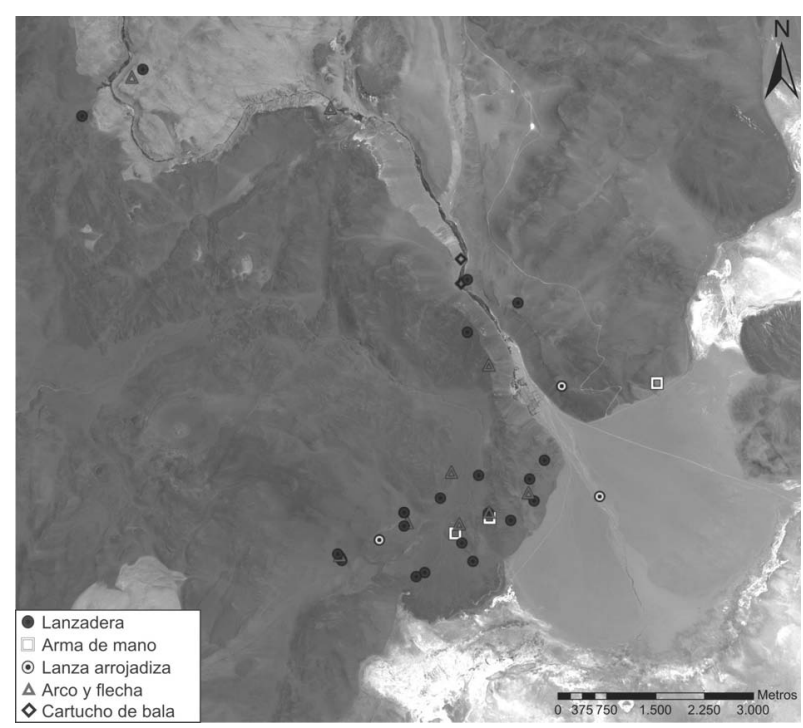

Figura 5. Distribución espacial de las puntas de proyectil en el sector de la desembocadura de la quebrada y el Campo de Antofalla de acuerdo al tipo de arma.

Figure 5. Spatial distribution of projectile points registered in the lower section of the Antofalla valley and the Campo Antofalla in accordance with the type of weapons.

a distintos momentos históricos ocupando los mismos espacios, es decir aquellos interpretados como relevantes a la hora de realizar la cacería, hablamos entonces de abras, pasos o quiebre de pendientes donde también fueron construidas estructuras para aumentar el escondite de los cazadores (Figura 6). Salvo en el área cercana al paleocauce en el cono de deyección que forma el denominado "Campo de Antofalla", donde se puede observar solamente especímenes correspondientes al período de "transición". A partir de esta información es posible interpretar la inexistencia de diferencias significativas en torno al aprovechamiento de espacios diversos en momentos históricos específicos.

\section{Tecnología, paisajes y cazadores}

En la quebrada de Antofalla, hemos registrado una variedad de diseños de puntas de proyectil, que se relaciona con el uso de distintos tipos de armas y contextos históricos variados, utilizados para la apropiación de camélidos silvestres. Pero más allá de esta variabilidad a través del tiempo, observamos la utilización de materias primas locales y técnicas de tallado que se reproducen en el tiempo.

Los diseños de las puntas de proyectil constituyeron las partes activas de distintos tipos de armas, donde hemos podido diferenciar el uso de propulsor, arco y flecha, lanza arrojadiza y armas de mano. Las características tecnológicas de estas armas, sumadas a las particularidades etológicas de las presas pretendidas habrían hecho necesaria la obtención de blancos relativamente cercanos para poder obtener los recursos

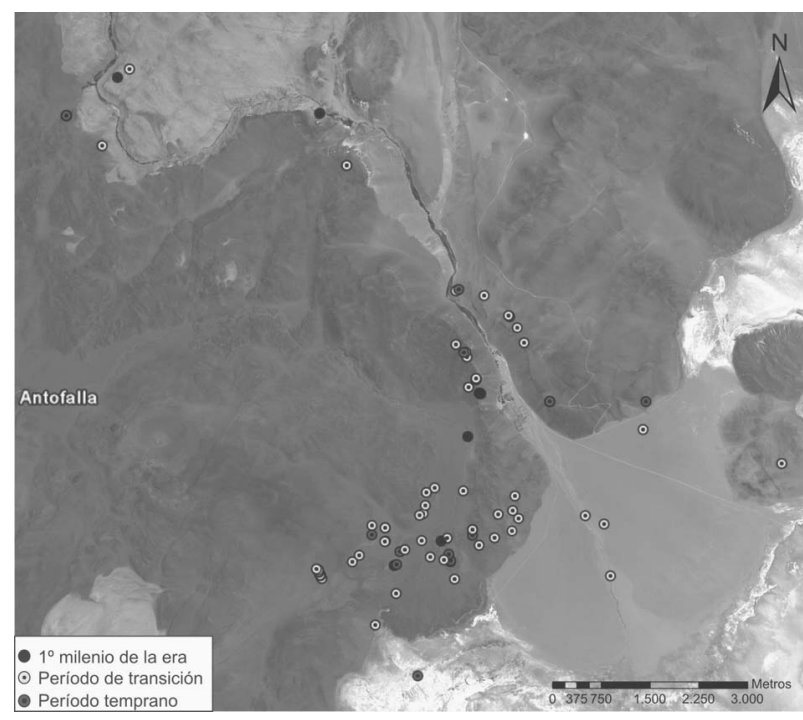

Figura 6. Distribución espacial de las puntas de proyectil en el sector de la desembocadura de la quebrada y el Campo de Antofalla de acuerdo a su asignación cronológica relativa.

Figure 6. Spatial distribution of projectile points registered in the lower section of the Antofalla valley and the Campo de Antofalla in accordance with the relative chronological assignment.

deseados. En este sentido, la estructuración de los paisajes de cacería, a través de la construcción de estructuras de diversa funcionalidad (tales como escondite, refugio o demarcación) en posiciones topográficas significativas, resulta necesaria para la posibilidad de obtener los recursos pretendidos (Moreno 2009, 2010b). Esta situación se encuentra también apoyada en la sistemática reutilización de los mismos espacios a través del tiempo y con los diferentes tipos de armas que fueron utilizados por las poblaciones humanas que ocuparon la quebrada de Antofalla en diferentes contextos históricos. Es claro que es necesario profundizar a través de la articulación de otras fuentes de datos las estrategias de caza y sus posibles cambios o continuidades en relación con las armas utilizadas (Aschero y Martínez 2001) e incluso con las especies pretendidas (Pintar 2008). Este es un camino sobre el cual continuar estas investigaciones.

En resumen, las prácticas de cacería de camélidos silvestres en la quebrada de Antofalla serían relevantes para la obtención de distintos tipos de recursos (carne, lana, tendones, cueros, etc.), donde la articulación entre las características etológicas de las presas pretendidas, las características de la tecnología de cacería y la estructuración de los paisajes, a través de la construcción de estructuras con diferentes funcionalidades, habría sido necesaria para alcanzar el éxito pretendido.

Catamarca, 15 de septiembre de 2010.

\section{Agradecimientos}

A Alejandro Haber, por el apoyo en las investigaciones 
en la quebrada de Antofalla. A todas las personas que participaron de la prospección en Antofalla, especialmente a Leandro D'Amore, Enzo Acuña, Patricia Lobo y Cecilia Cuello. A Jorge Martínez, Salomón Hocsman y Carlos Aschero, por la colaboración en la comparación de los diseños de las puntas de proyectil. A Patricia Escola que también fue importante en el análisis de estos instrumentos. A Lizzie Pintar por el valioso aporte de bibliografía. A los evaluadores, por los enriquecedores comentarios al texto. $Y$ especialmente a los pobladores de Antofalla por permitirme trabajar en sus tierras.

\section{Bibliografía}

Aguerre, A., A. Fernández Distel y C. Aschero. 1973. Hallazgo de un sitio acerámico en la Quebrada de Inca Cueva (Pcia. De Jujuy). Relaciones de la Sociedad Argentina de Antropología VII: 197-235.

Ambrosetti, J. 1976. Supersticiones y leyendas. Ediciones Siglo Veinte. Buenos Aires.

Aschero, C. 1975. Ensayo para una clasificación morfológica de artefactos líticos aplicada a estudios tipológicos comparativos. Informe presentado al Consejo Nacional de Investigaciones Científicas y Técnicas (CONICET). M.S.

Aschero, C. 1983. Ensayo para una clasificación morfológica de artefactos líticos. Apéndice A y B. Cátedra de Ergología y Tecnología. Facultad de Filosofía y Letras. Universidad de Buenos Aires. MS.

Aschero, C., D. Elkin y E. Pintar. 1991. Aprovechamiento de recursos faunísticos y producción lítica en el precerámico tardío. Un caso de estudio: Quebrada Seca 3 (Puna Meridional Argentina). Actas del XI Congreso Nacional de Arqueología Chilena Tomo II: 101-114. Museo Nacional de Historia Natural, Santiago de Chile.

Aschero, C. y J. Martínez. 2001. Técnicas de caza en Antofagasta de la Sierra, Puna meridional, Argentina. Relaciones de la Sociedad Argentina de Antropología XXVI: 215-241.

Binford, L.R. 1972. An Archaeological Perspective. Seminar Press, New York, NY

Bleed, P. 1986. The optimal design of hunting weapons: maintanibility or realibility. American Antiquity 51(4): 737-747.

Bonacic, C. 2005. Vicuña ecology and management. International Camelid Quarterly 4(4): 75-82. Rysko Pearson, Canada.

Cabrera, A. 1957. La vegetación de la Puna argentina. Revista de investigaciones agrícolas 11(1): 317-413.
Cartajena, I. 1994 Determinación de restos óseos de camélidos en dos yacimientos del Loa Medio (II Región). Estudios Atacameños 11:25-52.

Cartajena, I. 2003. "Los conjuntos arqueofaunísticos del arcaico temprano en la Puna de Atacama, Norte de Chile". Tesis Doctoral. Freie Universität Berlin. Impresa en microfilm. ABESY Vertriebs GmbH, Alemania.

Cattelain, P. 1997. Hunting during the Upper Paleolithic: bow, spearthrower, or both? En H. Knecht (ed.). Projectil Technology, pp: 213-240. Plenum Press, New York.

Churchill, S. 1993. Weapon technology, prey size selection, and hunting methods in modern huntersgatherers: implications for hunting in the Palaeolithic and Mesolithic. Archaeological Papers of the American Anthropological Association 4: 11-24.

Cieza de León, P. [1553] 1984. La crónica del Perú. Edición de Manuel Ballesteros. Historia 16. Madrid.

Cotterell, B. Y J. Kamminga. 1990. Mechanics of Preindustrial Technology: an introduction to the mechanics of ancient and traditional material culture. Cambridge University Press. Cambridge.

Criado Boado, F. 1999. Del Terreno al Espacio: planteamientos y perspectivas para la arqueología del paisaje. Capa 6.

Custred, G. 1979. Hunting technologies in Andean Culture. Journal de la Societe des Americanistes. Tome LXVI: 7-19. París.

Dedenbach, S. 1990. La caza de los camélidos silvestres. Inka Pachaq Llamanpa Willaynin. Uso y crianza de los camélidos en la época incaica. Bonner Amerikanistische Studien 16. Bonn.

De Souza, P. 2004. Tecnologías de proyectil durante los períodos Arcaico y Formativo en el Loa Superior (Norte de Chile) a partir del análisis de puntas líticas. Chúngara. Volumen especial. Tomo I: 61-76.

Dobres, M. 2000. Technology and Social Agency. Blackwell Publishers. Oxford

Edmonds, M. 1995. Stone tools and society. Working stone in Neolithic and Bronze Age Britain. BT Batsford, Londres.

Elkin, D. 1992. Disponibilidad y explotación de recursos en relación al sitio acerámico Quebrada Seca 3, Antofagasta de la Sierra, Puna de Catamarca. Shincal 2: 1-14. Escuela de Arqueología, Universidad Nacional de Catamarca.

Elkin, D. 1996. “Arqueozoología de Quebrada Seca 
3: indicadores de subsistencia temprana, en la Puna Meridional Argentina". Tesis doctoral inédita, Facultad de Filosofía y Letras, Universidad de Buenos Aires, Argentina.

Escola, P. 1987. "Las puntas de proyectil del formativo en Puna y Quebradas de Acceso: un estudio tecno-tipológico de cuatro casos de análisis". Tesis de Licenciatura inédita. Facultad de Filosofía y Letras, Universidad de Buenos Aires, Argentina.

Escola, P. 2000. "Tecnología lítica y sociedades agropastoriles tempranas". Tesis Doctoral inédita. Facultad de Filosofía y Letras. Universidad Nacional de Buenos Aires. Argentina.

Fenenga, F. 1953. The weights of chipped stone points: A clue to their functions. Southwestern Journal of Anthropology 9: 309-323.

Fernández Distel, A. 1986. Las cuevas de Huachichocana, su posición dentro del precerámico con agricultura incipiente del Noroeste Argentino. Beitrage zur Allgemeinen und Vergleichenden Archaeologie, band 8: 353-430. Manz am Rehein

Franklin, W. 1982. Biology, Ecology, and Relationship to man of the South American Camelids. En Mammalian Biology in South American, editado por M. Mares, y $\mathrm{H}$. Genoways, pp.457-490. University of Pittsburgh Press, Pittsburgh.

Gambier, M. 1985. La cultura de Los Morrillos. Instituto de Investigaciones Arqueológicas y Museo. Facultad de Filosofía, Humanidades y Artes. Universidad Nacional de San Juan.

García, S., D. Rolandi y D. Olivera. 2000 Puna e historia. Antofagasta de la Sierra, Catamarca. Tridente, Asociación Amigos del Instituto Nacional de Antropología, Buenos Aires.

Gero, J. 1989. Assessing social information in material objects: how well do lithics measure up? En R. Torrence (Ed.). Time, energy and stone tools, pp: 92-105. Cambridge University Press. Cambridge.

Gianotti, C. 2004. La prospección como estrategia metodológica para el estudio del paisaje monumental en las tierras bajas uruguayas. En Arqueología Espacial 23-24, Arqueología espacial: Prospección, coordinado por F. Burillo, pp: 259-282.

Haber, A. 1991. Investigaciones metodológicas en arqueología: el caso del pastoreo de llamas. Cuaderno de Humanidades 5:69-81. Salta.

Haber, A. 1999. Informe de evaluación de impacto arqueológico de la fase de exploración de la reserva minera Antofalla Este. Centro Editor. Universidad Nacional de Catamarca.

Haber, A. 2003a. Hunting after Domestication. Trabajo presentado en CHAGS 9. Edimburgo.

Haber, A. 2003b. Informe presentado a la National Geographic Society. MS.

Haber, A. 2006. Una arqueología de los paisajes puneños. Domesticidad, interacción e identidad en Antofalla. Primer y segundo milenios d.C. Jorge Sarmiento Editor. Universitas Libros, Córdoba.

Haber, A. 2007. Vicuñas and domesticity. En M. Gutiérrez, L. Miotti, G. Barrientos, G. Mengoni Goñalons y M. Salemme (eds.). Taphonomy and zooarchaeology in Argentina, pp: 59-70. BAR International Series 1601. Archaeopress. Oxford.

Haber, A. 2009. Animism, Relatedness, Life: Post-Western Perspectivas. Cambridge Archaeological Journal 19(3): 418-430

Haber, A., M. Gastaldi y M. Quesada. 2002 Arqueología industrial de un enclave minero salteño en Bolivia, mediados del siglo XIX. Actas de las XVIII Jornadas de Historia Económica. Mendoza. CD-Rom.

Haber, A. y E. Moreno. 2008. Dos veces en la misma trampa. Notas de arqueología casi crepuscular. Trabajo presentado en las Jornadas de Arqueología del Área Puneña de los Andes Centro-Sur. Tendencias, variabilidad y dinámicas de cambio (ca. 11000-1000 AP). Horco Molle. Tucumán.

Hocsman, S. 2006. "Producción lítica, variabilidad y cambio en Antofagasta de la Sierra -ca. 5500 - 1500 AP". Tesis doctoral inédita. Facultad de Ciencias Naturales y Museo. Universidad Nacional de La Plata, Argentina.

Hoffmann, E., Otte, K., Ponce, C. y Ríos, M. 1983. El manejo de la vicuña silvestre. Tomo II. Eschborn. Sociedad Alemana de Cooperación Técnica (GTZ).

Hughes, S. 1998. Getting to the point. Evolutionary change in prehistoric weaponry. Journal of Archaeological Method and Theory. Vol 5(4): 345-408. Nueva York y Londres. Plenum Press.

Jofré, I. 2004. "Arqueología del fuego. Tebenquiche Chico". Tesis de Licenciatura inédita. Escuela de Arqueología. Universidad Nacional de Catamarca.

Kidder, A. 1938. Arrow-heads or dart points. American Antiquity 4: 156-157

Koford, C. 1957. The vicuña and the puna. Ecological 
monographs Vol. $27 \mathrm{~N}^{\circ} 2$. Museum of Vertebral Zoology. University of California.

Lema, C. 2004. "Tebenquiche Chico en los siglos XVI y XVII". Tesis de Licenciatura inédita. Escuela de Antropología, Facultad de Humanidades y Artes, Universidad Nacional de Rosario, Argentina.

Lema, C. y E. Moreno. 2009. De la puna al mercado. La caza de vicuñas en el contexto colonial. Trabajo presentado en las XII Jornadas Interescuelas/Departamentos de Historia. San Carlos de Bariloche.

López, G. 2003 Pastoreo y caza de camélidos en el Temprano de la Puna de Salta: Datos osteométricos del sitio Matancillas 2. Intersecciones en Antropología 4: 17-27.

López, G. 2006 Resultados del análisis arqueofaunístico de un basural, un recinto y un sector interrecinto del sitio Matancillas 2, Puna de Salta. Intersecciones en Antropología 7:207-216.

Martínez, J. 2003. “Ocupaciones humanas tempranas y tecnología de caza en la microrregión de Antofagasta de la Sierra (10000-7000 AP)". Tesis doctoral inédita. Facultad de Ciencias Naturales e Instituto Miguel Lillo. Universidad Nacional de Tucumán, Argentina.

Martínez, J. 2005. Tecnología de cazadores en la Puna Meridional Argentina: el caso de Peñas de la Cruz 1. Mundo de Antes 4, 25-49.

Martínez, J., M. Mondini, E. Pintar y M. C. Reigadas 2010. Cazadores-recolectores tempranos de la Puna Merdidional Argentina: avances en su estudio en Antofagasta de la Sierra (Pleistoceno final-Holoceno temprano/medio). En J. R. Bárcena y H. Chiavazza (eds.), Arqueología Argentina en el Bicentenario de la Revolución de Mayo. XVII Congreso Nacional de Arqueología Argentina: 16911696. Mendoza, FFyL UNCU-INCIHUSA CONICET.

Millones, L. y R. Schaedel. 1980. Plumas para el sol: Comentarios a un documento sobre cazadores y cotos de caza en el antiguo Perú. Bulletin del Instituto Francés de Estudios Andinos IX, No1-2: 59-88.

Mondini, M. y D. Elkin. 2006. Cazadores-Recolectores de la Cuenca de Antofagasta de la Sierra (Puna Meridional Argentina): Una perspectiva zooarqueológica y tafonómica. Cazadores y Recolectores del Cono Sur. Revista de Arqueología 1: 67-79.

Mondini, M., M. Reigadas, E. Pintar y J. Martínez. 2011. Cazadores-recolectores de Antofagasta de la Sierra en el Holoceno Medio. En M. Mondini, J. Martínez, H. Muscio y B. Marconetto (eds.) Poblaciones humanas y ambientes en el Noroeste Argentino durante el Holoceno Medio:

\section{7-82. Gráfica Corintios 13. Córdoba.}

Moreno, E. 2005. "Artefactos y prácticas. Análisis tecnofuncional de los materiales líticos de Tebenquiche Chico 1". Tesis de Licenciatura inédita. Escuela de Arqueología. Universidad Nacional de Catamarca, Argentina.

Moreno, E. 2006. Tecnología lítica y agentes sociales en Tebenquiche Chico. Aportes científicos desde Humanidades 6: 241-251.

Moreno, E. 2009. El paisaje cazador en la quebrada de Antofalla. Antofagasta de la Sierra, Catamarca. La Zaranda de Ideas 5: 101-120.

Moreno, E. 2010a. Armas en el paisaje. Aportes metodológicos para su estudio. Trabajo presentado en el XVII Congreso Nacional de Arqueología Argentina. Mendoza.

Moreno E. 2010b. "Arqueología de la caza de vicuñas en el área del Salar de Antofalla, Puna de Atacama. Una aproximación desde la arqueología del paisaje". Tesis doctoral inédita. Facultad de Ciencias Naturales y Museo. Universidad Nacional de La Plata, Argentina.

Moreno E. y C. Revuelta. 2010. La caza de vicuñas en Tebenquiche Chico (Dpto. Antofagasta de la Sierra, Catamarca). Un acercamiento de larga duración. Relaciones de la Sociedad Argentina de Antropología volumen XXXV: 171-194.

Morlans, M. 1995. Regiones naturales de Catamarca. Provincias geológicas y provincias fitogeográficas. Revista de Ciencia y Técnica 2: 1-36.

Nance, J. 1979. Regional subsampling and statistical inference in forested habitats. American Antiquity 44(1): 172-176.

Nuñez, L. 1983. Paleoindio y arcaico en Chile: Diversidad, secuencias y procesos. Cuicuilco. México.

Núñez, L y M. Santoro. 1988. Cazadores de la puna seca y salada del área centro-sur andina (norte de Chile). Estudios Atacameños 9: 11-60.

Núñez, L., M. Grosjean e I. Cartajena. 2005. Ocupaciones humanas y paleoambientes en la Puna de Atacama. Instituto de Investigaciones Arqueológicas y Museo. Universidad Católica del Norte. Taraxacum. San Pedro de Atacama.

Olivera, D. 1992. Tecnología y estrategias de adaptación en el Formativo (Agro-alfarero Temprano) de la Puna Meridional Argentina. Un caso de estudio: Antofagasta de la Sierra (Pcia. de Catamarca, R.A.). Tesis doctoral inédita. Facultad de Ciencias Naturales y Museo. 
Universidad Nacional de La Plata.

Olivera, D. 1997. La importancia del recurso Camelidae en la Puna de Atacama entre los 10.000 y 500 años A. P. Estudios Atacameños 14: 29-41.

Pfaffenberger, B. 1992. Social Anthropology of Technology. Annual Review of Anthropology 21: 491-516

Pintar, E. 1996. "Prehistoric Holocene adaptations to the Salt Puna of Northwest Argentina". Tesis doctoral inédita. Southern Methodist University, Dallas. Estados Unidos.

Pintar 2008. Astiles, intermediarios y sistemas de armas. Cazadores recolectores del Cono Sur, Revista de Arqueología 3: 115-128. Mar del Plata.

Polo de Ondegardo. [1571] 1990. El mundo de los Incas. Edición de Laura González y Alicia Alonso. Historia 16. Madrid.

Quesada, M. 2007. "Paisajes agrarios en el área de Antofalla. Procesos de trabajo y escalas sociales de la producción agrícola. Primer y segundo milenio d.C." Tesis doctoral inédita. Facultad de Ciencias Naturales y Museo, Universidad Nacional de La Plata.

Ratto, N. 1994. Funcionalidad versus adscripción cultural: cabezales líticos de la margen norte del estrecho de Magallanes. En J. Lanata y L. Borrero (eds.) Arqueología de Cazadores-recolectores. Límites, casos y aperturas, Arqueología Contemporánea 5: 105-120. Buenos Aires.

Ratto, N. 2003. "Estrategias de caza y propiedades del registro arqueológico en la Puna de Chaschuil (Departamento de Tinogasta, Catamarca, Argentina)". Tesis doctoral inédita. Facultad de Filosofía y Letras, Universidad de Buenos Aires, Argentina.

Reigadas. M. 1992. La punta del ovillo: determinación de domesticación y pastoreo a partir del análisis microscópico de fibras y folículos pilosos de camélidos. Arqueología 2: 9-52. Instituto de Ciencias Antropológicas. UBA. Buenos Aires.

Reigadas, M. 1994. Las fibras como indicador temprano del control cultural de camélidos en el NOA. Estudios Atacameños 11: 53-72.
Revuelta, C. 2005. "Apropiación social y vicuñas. Análisis zooarqueológico de la unidad doméstica TC1. Oasis de Tebenquiche Chico - Primer milenio d.C." Tesis de Licenciatura inédita. Escuela de Arqueología. Universidad Nacional de Catamarca.

Rodríguez, M. 1999. Sistemas de asentamiento y movilidad durante el Arcaico. Análisis de macrovestigios vegetales en sitios arqueológicos de la puna meridional argentina. Estudios Atacameños 14, 43 - 60.

Shott, M. 1997. Stone and shafts redux: the metric discrimination of chipped-stone dart and arrow points. American Antiquity 62(1): 86-101.

Thomas, D. 1978. Arrowheads and atlatl darts: how the stones got the shaft. American Antiquity 43: 461-472.

Tomka, S. 1992. Vicuñas and Llamas: Parallels in Behavioral Ecology and Implications for the Domestication of Andean Camelids. Human Ecology 20(4):407-433.

Troll, C. [1931]1980. Las culturas superiores andinas y el medio geográfico. Allpanchis phuturinqa XIV(15):3-55.

Wheeler, J. 2006. Historia natural de la vicuña. En Investigación, conservación y manejo de vicuñas, editado por B. Vilá, pp: 25-36. Proyecto MACS. Universidad Nacional de Luján.

Wiessner, P 1983. Style and social information in Kalahari San projectile points. American Antiquity 48(2): 253-276.

Yacobaccio, H y C. Madero. 1992. Zooarqueología de Huachichocana III (Jujuy, Argentina). Arqueología 2: 149-188.

Yacobaccio, H., D. Elkin y D. Olivera. 1994. ¿El fin de las sociedades cazadoras? El proceso de domesticación animal en los Andes Centro Sur. En J. Lanata y L. Borrero, (eds.). Arqueología de cazadores-recolectores. Límites, casos y aperturas, pp: 23-32. Arqueología contemporánea 5. Buenos Aires.

Yacobaccio, H., L. Killian y B. Vilá. 2007. La explotación de la vicuña durante el período colonial (1535-1810). El negocio de los cueros y lanas de vicuña. Todo es Historia $N^{\circ} 483$ : 16-21. 